\title{
Correction to: Identifying child temperament risk factors from 2 to 8 years of age: validation of a brief temperament screening tool in the US, Europe, and China
}

\author{
Marcel Zentner ${ }^{1}[$
}

Published online: 21 September 2019

๑) Springer-Verlag GmbH Germany, part of Springer Nature 2019

Correction to: European Child and Adolescent Psychiatry https://doi.org/10.1007/s00787-019-01379-5

Appendix excluded from article CC BY licence. All rights reserved.

The original article can be found online at https://doi.org/10.1007/ s00787-019-01379-5.

Marcel Zentner

Marcel.zentner@uibk.ac.at

1 Department of Psychology, University of Innsbruck, Innrain 52, 6020 Innsbruck, Austria 